\title{
Quality of antenatal care and associated factors in a rural county in Kenya: an assessment of service provision and experience dimensions
}

\author{
Patience A. Afulani ${ }^{1,2^{*}}$ (D), Laura Buback ${ }^{1,2}$, Francisca Essandoh ${ }^{3}$, Joyceline Kinyua ${ }^{4}$, Leah Kirumbi ${ }^{4}$ and
} Craig R. Cohen ${ }^{1,2}$

\begin{abstract}
Background: This study aimed to assess the quality of antenatal care (ANC) women received in Migori county, Kenya-including both service provision and experience dimensions - and to examine factors associated with each dimension.

Methods: We used survey data collected in 2016 in Migori county from 1031 women aged 15-49 who attended ANC at least once in their most recent pregnancy. ANC quality service provision was measured by nine questions on receipt of recommended ANC services, and experience of care by 18 questions on information, communication, dignity, and facility environment. We summed the responses to the individual items to generate ANC service provision and experience of care scores. We used both linear and logistic regression to examine predictors.

Results: The average service provision score was 10.9 (SD = 2.4) out of a total of 16. Most women received some recommended services once, but not at the frequency recommended by the Kenyan Ministry of Health. About $90 \%$ had their blood pressure measured, and $78 \%$ had a urine test, but only 58 and $14 \%$ reported blood pressure monitoring and urine test, respectively, at every visit. Only $16 \%$ received an ultrasound at any time during ANC. The average experience score is 27.3 ( $S D=8.2$ ) out of a total score of 42 , with key gaps demonstrated in communication. About half of women were not educated on pregnancy complications. Also, about one-third did not often understand the purposes of tests and medicines received and did not feel able to ask questions to the health care provider. In multivariate analysis, women who were literate, employed, and who received all their ANC in a health center had higher experiences scores than women who were illiterate (coefficient $=1.52, \mathrm{Cl}: 0.26,2.79$ ), unemployed (coefficient $=2.73, \mathrm{Cl}: 1.46,4.00$ ), and received some ANC from a hospital (coefficient $=1.99, \mathrm{Cl}$ : 0.84, 3.14) respectively. The wealthiest women had two times higher odds of receiving an ultrasound than the poorest women (OR $=2.00, \mathrm{Cl}: 1.20,3.33)$.

Conclusion: Quality of ANC is suboptimal in both service provision and experience domains, with disparities by demographic and socioeconomic factors and facility type. More efforts are needed to improve quality of ANC and to eliminate the disparities.
\end{abstract}

Keywords: Antenatal care, Quality of care, Person-centered care, Experience of care, Services provision, Kenya

\footnotetext{
* Correspondence: Patience.Afulani@ucsf.edu

'School of Medicine, University of California, San Francisco, USA

${ }^{2}$ Institute for Global Health Sciences, UCSF, San Francisco, USA

Full list of author information is available at the end of the article
}

(c) The Author(s). 2019 Open Access This article is distributed under the terms of the Creative Commons Attribution 4.0 International License (http://creativecommons.org/licenses/by/4.0/), which permits unrestricted use, distribution, and reproduction in any medium, provided you give appropriate credit to the original author(s) and the source, provide a link to the Creative Commons license, and indicate if changes were made. The Creative Commons Public Domain Dedication waiver (http://creativecommons.org/publicdomain/zero/1.0/) applies to the data made available in this article, unless otherwise stated. 


\section{Background}

Maternal and neonatal mortality has remained high in low resource settings despite progress in recent years. In 2015 , about 303,000 women died from pregnancy-related causes and 2.7 million babies died during the first 28 days of life globally [1, 2]. About 2.6 million babies were also stillborn [3]. High quality antenatal care (ANC) can reduce maternal and neonatal morbidity and mortality and stillbirths through prevention, as well as early identification and management of pregnancy complications or pre-existing conditions [4]. High quality ANC can also influence women's health seeking behavior towards choosing skilled care at birth and helping them prepare to be able to access it [5-7]. A positive experience during both pregnancy and childbirth are key to person-centered care and the right of every childbearing woman, as highlighted in recent World Health Organization (WHO) recommendations [8-10]. While the specific recommendations for frequency of ANC has varied, WHO has consistently recommended that all pregnant women receive some ANC during their pregnancy $[4,8,11]$.

Kenya's National Guidelines for Quality Obstetrics and Perinatal Care that were in use at the time of this work were based on the WHO recommendations on Focused Antenatal Care [12], which recommended four comprehensive and targeted visits. The guidelines, however, urged providers to view each visit as if it were the only visit a woman may make. The recommended content of each visit includes blood pressure and fetal growth monitoring, urine testing, Iron and Folic Acid supplementation, Tetanus Toxoid immunizations, at least two doses of Intermittent Preventative Treatment for malaria in pregnancy in endemic malaria areas and deworming after the first trimester. The first ANC visit also includes a more in-depth medical history and physical examination, including head to toe examination, recording weight and height, blood group typing, HIV testing and counseling, and assessing needs for specialized care [13]. The Kenya guidelines do not mention ultrasound scanning for routine ANC, but it is recommended for fetal assessment, including for dating, among women with preterm labor and those with complications. Early ultrasound increases the accuracy of gestational age assessments and current WHO guidelines recommend one ultrasound scan before 24 weeks of gestation [8].

The Kenya guidelines also recommend comprehensive health promotion education, with a question and answer session also recommended during each visit. These guidelines also emphasize the importance of patient experience components such as communication, respect, and dignity. It states that "Antenatal care should be simpler, safer, friendly and more accessible. Women are more likely to seek and return for services if they feel cared for and respected by their providers. This personalized approach requires health care providers to use excellent interpersonal skills since listening to client's concerns is just as important as giving advice. It respects clients' right to dignity, privacy, confidentiality, full and accurate information" [13]. Likewise, the most recent WHO recommendations on antenatal care for a positive pregnancy experience, updated in 2016, prioritizes person-centered care and overall well-being of the mother and baby [8].

Until recently, most prior research on maternal health care focused on use of services with research on ANC mostly on timing and frequency of ANC visits [14-17]. Increasing recognition of the role of poor-quality care to the poor maternal and neonatal outcomes has stimulated interest in assessing quality of maternal health service. However, most of the attention has focused on quality of care during childbirth. $[18,19]$. Little research thus exists on quality of ANC, and most of the studies on ANC quality have focused on the receipt of recommended ANC services [20-22]. This is despite a global movement advocating for measurement and interventions to improve respectful maternity care. Only a few studies in Kenya, based on the Service Provision Assessment data at the national level, have examined multiple dimensions of quality of care. These studies suggest sub-optimal ANC quality in structural, service provision, and experience measures [22-24].

More studies, including studies at sub-national levels examining multiple dimensions of ANC quality, are critical to (1) provide information on strengths and gaps in ANC quality and (2) guide interventions in specific areas to improve provision of services and person-centered antenatal care (PCANC). This paper extends the evidence in this area. We aimed to assess levels of quality of ANC, including both service provision and experience of care, in a rural county in western Kenya. Service provision here refers to receipt of recommended evidence-based services for ANC per WHO and Kenya guidelines. Experience of care captures items related to effective communication, respect, dignity, and emotional support per the WHO framework for quality of maternal and newborn health [10]. These experience dimensions assess PCANC. We also examine factors associated with each dimension of quality of care to identify sources of disparities in quality of care.

\section{Methods}

\section{Setting and data collection}

The data are from a cross-sectional survey conducted with women who had recently given birth as part of a study on community perceptions of quality of maternity care in Migori county. The setting and data collection methods have been previously described [25, 26]. Briefly, Migori County is a rural county in western Kenya with 8 
sub-counties and a population of about one million people [27]. The county has one referral hospital, seven sub-county hospitals, 18 health centers, several dispensaries, and a few faith-based and private health facilities [28]. The estimated total fertility rate for the county is 5.2 [29]. According to the 2014 Kenya Demographic and Health Survey, $96 \%$ of women with a live birth in the preceding five years received at least one ANC from a skilled provider, but only $56 \%$ received the recommended four-plus visits [29].

The survey was conducted in August and September 2016 with women aged 15-49 years who delivered in the nine weeks preceding the survey. A multistage sampling approach was used to select women from each subcounty in health facilities and in communities, with a target of interviewing at least 100 women from each sub-county. The interviews were conducted in English, KiSwahili, and DhLuo in private spaces in health facilities or in the homes of the respondents. About one thousand women $(N=1051)$ were interviewed, with a response rate above $98 \%$. Ethical approval for the study was provided by the institutions listed in the ethics statement, and all participants provided written informed consent after receiving information about the research. We use data from women who received $\mathrm{ANC}$ at least once during their most recent pregnancy $(N=1031)$. Women received ANC from government facilities (the referral hospital, health centers, and dispensaries) as well as private facilities.

\section{Measures \\ Dependent variables (outcomes): measures of quality of ANC}

Quality of ANC is measured by several questions asked to women to assess different aspects of the content of services they received and the nature of their interactions with providers during ANC visits (Additional file 1). The measures explored capture of both service provision and experience of care.

Service provision The service provision measures are nine items asking whether they received various services for screening and prevention listed in Table 2 . The questions include whether they had their height, weight, and blood pressure measured, whether they had urine and blood tests, and whether they received tetanus injections, iron supplements, antihelminthes, and antimalarials. These questions were adapted from the ANC questions in the Demographic and Health Surveys. Five of the questions have binary responses $(\mathrm{No}=0$ and $\mathrm{Yes}=1)$; three have responses on a four-point frequency scale (No never $=0$, Yes, a few times $=1$, and Yes, most of the time $=2$, and Yes, all the time $=3$ ); and one is on a three-point frequency scale $((\mathrm{No}=0$, Yes, once $=1$, and Yes, more than once $=2$ ). These items have factor loadings of $>.2$ on one factor and a reasonable Cronbach alpha of 0.5. To examine the associated factors, the response codes for the nine items are summed to create a service provision index. The summative score has a range of 0 to $16\left(\left(5^{*} 1\right)+\left(3^{*} 3\right)+\left(1^{*} 2\right)\right)$. 'Don't know' response options are recoded to missing before summing the responses. Ultrasound screening is examined separately as a binary variable because it was not routinely recommended and did not load well with the rest of the items.

Experience of care The experience of care measures are also listed in Table 3 . These questions capture mostly information sharing and communication: told results after she has been weighed, her blood pressure taken, and after urine and blood tests; educated on pregnancy complications, what to do in the event of a complication, what to expect during the pregnancy, birth preparedness, diet, and breastfeeding. Furthermore, they asked whether she understood the purpose of tests and medicines, whether she was asked if she had questions, and if she felt able to ask questions. There are also three questions capturing dignity and respect as well as one question on cleanliness of the facility. These questions were adapted from the ANC questions in the Demographic and Health Surveys, as well as from the person-centered maternity care scale [26]. Six of the questions have binary responses $(\mathrm{No}=0$ and $\mathrm{Yes}=1)$, and 12 have responses on a four-point frequency scale (No never $=0$, Yes, a few times $=1$, and Yes, most of the time $=2$, and Yes, all the time $=3$ ). The items load together on one factor with loadings greater than 0.3 and have Cronbach alpha of 0.81 . The response codes to the 18 items are summed to create an experience of care index to examine associated factors. The summative score has a range of 0 to 42 $\left(\left(6^{*} 1\right)+(12 * 3)\right)$. 'Don't know' response options are recoded to missing before summing the responses. We also included two items (being asked for bribe and feeling they were treated differently based on certain attributes) to assess predictability and transparency of payments and perceptions of discrimination. However, these two are not included in the index because they loaded poorly with the rest of the items in the group in the factor analysis.

\section{Independent variables (predictors)}

Based on prior studies and theoretical rationale, we examined various factors that might affect the quality of ANC a woman receives, including: socioeconomic and demographic factors, women's health status, familiarity and extent of contact with the health system, and facility and provider characteristics. The demographic factors included are age, marital status, parity, tribe, and religion [20-23]. 
The key independent variables are the socioeconomic factors which capture a woman's personal status and empowerment and her status based on her household or partner. These included Employment status, type of Occupation, Education, and Literacy, which capture economic empowerment (access to and control over the means to make a living, and receiving the material benefits of this access), cognitive and psychological empowerment (includes knowledge about rights, self-esteem, and self-efficacy) [30-32]. We also included two composite measures of empowerment: participation in household decision-making and attitudes towards domestic violence to measure sociocultural empowerment (gender norms, including norms against gender-based violence) [25, 33]. We hypothesized that more empowered women will receive higher quality care. In addition, we included experience of domestic violence, which may be associated with both empowerment and mistreatment [25, 34]. Other measures of socioeconomic status (SES) included are Household wealth (measured in quintiles, calculated from a wealth index based on 13 questions on household assets [35] and Partner's education and occupation. In addition, we included a variable on whether they or someone in their household works in a health facility, as this could influence the type of care they receive.

Variables to capture health status related factors that might affect the care women receive include whether they had complications in the index or prior pregnancy and their own assessment of the severity of the complications (whether they felt the complication was severe or not), as well as reason for seeking ANC (for a problem or checkup). For familiarity with and extent of contact with the health system, we included a variable on whether they had received ANC in prior pregnancy, whether they had previously delivered in a health facility, and the timing and frequency of ANC. For facility and provider characteristics, we included two variables on the type of facility the woman received care in and the type of provider. Because a woman could receive care from more than one type of facility and provider, these were recoded into the highest type of facility and provider (e.g., if they received care from a hospital and a health center, it is coded as hospital, and if they received care from a doctor and nurse, it is coded as a doctor). Finally, we controlled for the timing and setting of the interview, as this might affect their responses.

\section{Analysis}

We first ran descriptive analysis to examine the characteristics of the sample and the distribution of all ANC quality related variables using means for continuous variables and proportions for categorical variables. Next, we assessed which of the ANC quality related variables could be grouped together to generate the ANC service provision and experience of care indices using exploratory factor analysis and Chronbach's alpha for internal consistency. We then summed the selected indices to generate ANC service provision and experience of care scores, which we used as the outcome variables in bivariate and multivariate analysis.

Because the summative scores are approximately normally distributed, we used them as continuous variables and examined mean differences in scores by the various predictors, as well as unadjusted and adjusted ordinary least squares (linear) regressions. The coefficients from the linear regressions quantify the change in scores for each unit change in continuous predictors or the difference in scores between the reference category and other categories for categorical predictors [36]. We used logistic regressions to examine the factors associated with receipt of an ultrasound. Given ultrasound is recommended for women with complications, we were particularly interested in whether women with complications, as well as certain risk factors are more likely to get an ultrasound. In addition, we dichotomized the service provision and experience of care scores around the median into low and high quality and examined them in logistic regressions for sensitivity analysis. For the multivariate analysis, we first included the key independent variables, then all of the variables that were significantly associated with the outcome measures in the bivariate analysis, in the multivariate models. We then conducted post estimation tests to assess model fit and checked for collinearity and removed variables that did not improve the models or were highly correlated with other variables in the model.

\section{Results}

\section{Descriptive}

Table 1 shows the characteristics of the sample. The average age was 25 years, and about $17 \%$ were less than 20 years old. Approximately 79\% were married, with average parity of $3 ; 30 \%$ had 4 or more children. About $60 \%$ had only primary education or less and $76 \%$ were literate (could read and write very well). Less than a quarter $(23 \%)$ were gainfully employed (work for which they were paid). About two-thirds started ANC in the second trimester and received more than four ANC visits. Most women received ANC from a nurse or midwife $(88 \%)$ and solely from a health center or dispensary (55\%). About $10 \%$ received ANC solely from a private facility and 34\% received some ANC from a hospital. Eighty-eight percent went for their first ANC visit for a checkup (routine ANC), but 46\% experienced some complication during the pregnancy and $31 \%$ felt the problem they had was severe. Table 2 and Table 3 shows the distribution of individual ANC quality measures for both service provision and experience of care. 
Table 1 Sample distribution

Age
15 to 19 years
20 to 29 years
30 to 48 years
Current marital status ${ }^{a}$
Single
Partnered/Cohabiting
Married
Widowed
Divorced/Separated

Number of births ${ }^{\mathrm{a}}$

1
2
3
4 or more
Highest education
No school/Primary
Post-primary/vocational/Secondary
College or above

Literacy: reading and write very well

$\begin{array}{ll}\text { No } & 244 \\ \text { Yes } & 787\end{array}$

Employed with income

No
Yes

Self or household member work in health facility

$\begin{array}{ll}\text { No } & 967 \\ \text { Yes } & 64\end{array}$

Household wealth quintile ${ }^{a}$

Poorest

Poorer

Middle

Richer

Richest

Household wealth quintile

Poorest/Poorer

Middle

Richer/Richest

Current occupation

Agricultural labor

Casual labor

Salaried worker

Self-employed in petty trade

Self-employed small-scale industry

Unemployed/homemaker

Other

No. $\%$

177

599

24.2

37.7

18.3
Table 1 Sample distribution (Continued)

\begin{tabular}{|c|c|c|}
\hline & No. & $\%$ \\
\hline \multicolumn{3}{|l|}{ Partner's occupation ${ }^{\mathrm{a}}$} \\
\hline Agricultural labor & 213 & 20.7 \\
\hline Casual labor & 185 & 18 \\
\hline Salaried worker & 157 & 15.3 \\
\hline Self-employed in petty trade & 144 & 14 \\
\hline Self-employed small-scale industry & 85 & 8.3 \\
\hline Unemployed/homemaker & 25 & 2.4 \\
\hline Other & 4 & 0.4 \\
\hline No Partner & 215 & 20.9 \\
\hline \multicolumn{3}{|l|}{ Partner's education ${ }^{a}$} \\
\hline No school/Primary & 397 & 39.3 \\
\hline Post-primary/vocational/Secondary & 250 & 24.8 \\
\hline College or above & 147 & 14.6 \\
\hline No Partner & 215 & 21.3 \\
\hline \multicolumn{3}{|l|}{ Has health insurance ${ }^{a}$} \\
\hline No & 866 & 84.2 \\
\hline Yes & 162 & 15.8 \\
\hline \multicolumn{3}{|l|}{ Tribe $\mathrm{a}^{\mathrm{a}}$} \\
\hline Luo & 696 & 67.6 \\
\hline Kuria & 239 & 23.2 \\
\hline Other & 95 & 9.2 \\
\hline
\end{tabular}

Religious affiliation

$\begin{array}{lll}\text { Catholic } & 271 & 26.3\end{array}$

Protestant/Pentecostal

Muslim/other religion

Attitude towards domestic violence

Tolerant

47.5

Intolerant

Participation in household decisions

Low participation

High participation

Experienced domestic violence

No

47.3

Yes

Had any pregnancy complications

No

54.2

Yes

45.8

16.5

Had severe pregnancy complications

No

709

Had complications in prior pregnancy 
Table 1 Sample distribution (Continued)

\begin{tabular}{|c|c|c|}
\hline & No. & $\%$ \\
\hline No & 339 & 32.9 \\
\hline Yes & 692 & 67.1 \\
\hline \multicolumn{3}{|l|}{ Prior facility delivery } \\
\hline No & 398 & 38.6 \\
\hline Yes & 633 & 61.4 \\
\hline \multicolumn{3}{|l|}{ Highest ANC facility } \\
\hline Gov't Hospital & 354 & 34.3 \\
\hline Gov't HC/Dispensary & 571 & 55.4 \\
\hline Mission/Private facility & 106 & 10.3 \\
\hline \multicolumn{3}{|l|}{ Highest ANC Provider type } \\
\hline Nurse/Midwife & 905 & 87.8 \\
\hline Doctor/Clinical officer & 115 & 11.2 \\
\hline Non-skilled attendant & 11 & 1.1 \\
\hline \multicolumn{3}{|l|}{ Reason for first $\mathrm{ANC}^{\mathrm{a}}$} \\
\hline Because of a problem & 112 & 10.9 \\
\hline Just for a checkup & 909 & 88.3 \\
\hline Can't Remember & 9 & 0.9 \\
\hline \multicolumn{3}{|l|}{ Timing of first antenatal visit } \\
\hline First trimester & 300 & 29.1 \\
\hline Second trimester & 634 & 61.5 \\
\hline Third Trimester & 97 & 9.4 \\
\hline \multicolumn{3}{|l|}{ Number of antenatal visits ${ }^{a}$} \\
\hline Less than 4 & 368 & 35.8 \\
\hline 4 or 5 & 547 & 53.3 \\
\hline 6 plus & 112 & 10.9 \\
\hline \multicolumn{3}{|l|}{ Place of interview } \\
\hline Health facility & 421 & 40.8 \\
\hline In the community/a home & 610 & 59.2 \\
\hline \multicolumn{3}{|l|}{ Postpartum length } \\
\hline less than 1 week & 81 & 7.9 \\
\hline 1 week or more & 950 & 92.1 \\
\hline $\mathrm{N}$ & 1031 & 100 \\
\hline
\end{tabular}

Service provision About $60 \%$ had their height measured during their ANC and 80\% reported their weight was measured at every visit. While guidelines recommend blood pressure and urine test at every ANC visit, only $58 \%$ reported their blood pressure was taken at every visit, and only $14 \%$ reported a urine test at every visit. Almost all (97\%) received a blood test at least once, with $20 \%$ receiving a blood test more than once. In terms of preventative interventions, about $87 \%$ received a tetanus injection, $89 \%$ received iron supplementation, 58\% received deworming medicine, and $82 \%$ were given antimalarial drugs, all of which are recommend per Kenya and WHO ANC guidelines. Only 16\% of the women received an ultrasound during ANC. The average service provision score is $10.9(\mathrm{SD}=2.4$; range $=1$ to 16$)$ out of 16.

Experience of care Women were not consistently informed of the results of their examinations or about pregnancy and delivery: about $71 \%$ reported they were always told their results after weighing, 53\% always told blood pressure results, $38 \%$ always told urine test results, and $56 \%$ always told blood test results. Similarly, only $47 \%$ were told about the signs of pregnancy complications, $57 \%$ told where to go in case of complications, and $44 \%$ told what to expect during pregnancy and delivery. Over three-quarters (77\%) reported receiving birth preparedness education, $67 \%$ nutrition education, and $64 \%$ breastfeeding education.

At least one out of three women reported sub-optimal understanding of ANC procedures: about 66\% understood the purposes of tests performed most or all of the time and $68 \%$ understood the purposes of medicines received most or all of the time. Less than two-thirds of women $(61 \%)$ felt they were able to ask questions most or all the time, and only $50 \%$ were consistently (most or all the time) asked if she had any questions.

Most women felt they were treated with respect most or all of the time $(90 \%)$, and about $87 \%$ felt they were treated in a friendly manner most or all of the time. However, in terms of confidentiality, about $31 \%$ reported they could never discuss issues in private. Over half of the women $(58 \%)$ felt the health facility was always clean. The majority of women were never asked to give a bribe (89\%) and felt they were never treated differently because of any personal attribute (94\%). These two measures were not included in the experience score index. The average experience score is $27.3(\mathrm{SD}=8.2$; range $=1$ to 42 ) out of 42 . The average score limited to the communication related items (excluding four questions on dignity and cleanliness) is $18.2(\mathrm{SD}=6.8$; range $=1$ to 30) out of 30.

\section{Bivariate}

Table 4 shows bivariate statistics for the association between the summative ANC quality measures and receipt of an ultrasound with various potential predictors. Significant differences exist in the ANC quality measures by sociodemographic factors as well as facility types. The following associations had $p$-values $<0.05$. Not accounting for other factors, women who were older than 19 years and married women had, on average, higher experience of care scores than younger and unmarried women, respectively. Older women were also more likely to receive an ultrasound examination than younger women. Women with higher parity had lower service provision scores, including less likely to get an ultrasound. 
Table 2 Distribution of quality of antenatal care variables

\begin{tabular}{|c|c|c|}
\hline Service provision & No. & $\%$ \\
\hline \multicolumn{3}{|l|}{ Height measured } \\
\hline No & 406 & 39.4 \\
\hline Yes & 616 & 59.7 \\
\hline Don't know or can't remember & 9 & 0.9 \\
\hline \multicolumn{3}{|l|}{ Weighed } \\
\hline No, Never & 22 & 2.1 \\
\hline Yes, A Few Times & 93 & 9 \\
\hline Yes, Most Of The Time & 86 & 8.3 \\
\hline Yes, All The Time & 828 & 80.3 \\
\hline Don't know or can't remember & 2 & 0.2 \\
\hline \multicolumn{3}{|l|}{ Blood pressure taken } \\
\hline No, Never & 99 & 9.6 \\
\hline Yes, A Few Times & 207 & 20.1 \\
\hline Yes, Most Of The Time & 122 & 11.8 \\
\hline Yes, All The Time & 594 & 57.6 \\
\hline Don't know or can't remember & 9 & 0.9 \\
\hline \multicolumn{3}{|l|}{ Did urine test } \\
\hline No, Never & 223 & 21.6 \\
\hline Yes, A Few Times & 632 & 61.3 \\
\hline Yes, Most Of The Time & 31 & 3 \\
\hline Yes, All The Time & 139 & 13.5 \\
\hline Don't know or can't remember & 6 & 0.6 \\
\hline \multicolumn{3}{|l|}{ Did a blood test } \\
\hline No & 35 & 3.4 \\
\hline Yes, once & 789 & 76.5 \\
\hline Yes, more than once & 207 & 20.1 \\
\hline \multicolumn{3}{|l|}{ Received a tetanus injection } \\
\hline No & 130 & 12.6 \\
\hline Yes & 892 & 86.5 \\
\hline Don't know or can't remember & 9 & 0.9 \\
\hline \multicolumn{3}{|l|}{ Iron supplementation } \\
\hline No & 98 & 9.5 \\
\hline Yes & 915 & 88.7 \\
\hline Don't know or can't remember & 18 & 1.7 \\
\hline \multicolumn{3}{|l|}{ Antihelminthes } \\
\hline No & 379 & 36.8 \\
\hline Yes & 598 & 58 \\
\hline Don't know or can't remember & 54 & 5.2 \\
\hline \multicolumn{3}{|l|}{ Antimalarials $^{\mathrm{a}}$} \\
\hline No & 162 & 15.7 \\
\hline Yes & 849 & 82.4 \\
\hline Don't know or can't remember & 19 & 1.8 \\
\hline \multicolumn{3}{|l|}{ Ultrasound $d^{a}$} \\
\hline No & 862 & 83.7 \\
\hline Yes & 167 & 16.2 \\
\hline Don't know or can't remember & 1 & 0.1 \\
\hline Total & 1031 & 100 \\
\hline
\end{tabular}

Table 3 Distribution of quality of antenatal care variables

\begin{tabular}{|c|c|c|}
\hline Experience of care & No. & $\%$ \\
\hline \multicolumn{3}{|l|}{ Told the results after weighing ${ }^{\text {a }}$} \\
\hline No, Never & 103 & 10.2 \\
\hline Yes, A Few Times & 82 & 8.2 \\
\hline Yes, Most Of The Time & 104 & 10.3 \\
\hline Yes, All The Time & 711 & 70.7 \\
\hline Don't know or can't remember & 6 & 0.6 \\
\hline \multicolumn{3}{|c|}{ Told results after blood pressure measurements ${ }^{\mathrm{a}}$} \\
\hline No, Never & 187 & 20.3 \\
\hline Yes, A Few Times & 134 & 14.5 \\
\hline Yes, Most Of The Time & 90 & 9.8 \\
\hline Yes, All The Time & 493 & 53.4 \\
\hline Don't know or can't remember & 19 & 2.1 \\
\hline \multicolumn{3}{|l|}{ Told results after urine test ${ }^{a}$} \\
\hline No, Never & 115 & 14.4 \\
\hline Yes, A Few Times & 329 & 41.1 \\
\hline Yes, Most Of The Time & 46 & 5.7 \\
\hline Yes, All The Time & 303 & 37.8 \\
\hline Don't know or can't remember & 8 & 1 \\
\hline \multicolumn{3}{|l|}{ Told results after blood test ${ }^{\mathrm{a}}$} \\
\hline No, Never & 62 & 6.2 \\
\hline Yes, A Few Times & 304 & 30.5 \\
\hline Yes, Most Of The Time & 62 & 6.2 \\
\hline Yes, All The Time & 554 & 55.6 \\
\hline Don't know or can't remember & 14 & 1.4 \\
\hline \multicolumn{3}{|c|}{ Told about the signs of pregnancy complications ${ }^{a}$} \\
\hline No & 537 & 52.2 \\
\hline Yes & 485 & 47.1 \\
\hline Don't know or can't remember & 7 & 0.7 \\
\hline \multicolumn{3}{|c|}{ Told where to go in case of complications ${ }^{\mathrm{a}}$} \\
\hline No & 445 & 43.2 \\
\hline Yes & 582 & 56.5 \\
\hline Don't know or can't remember & 3 & 0.3 \\
\hline
\end{tabular}

Told what to expect during pregnancy and delivery

No 566

54.9

Yes 458

Don't know or can't remember

7

44.4

Birth preparedness education ${ }^{a}$

No $233 \quad 22.6$

$\begin{array}{lll}\text { Yes } & 793 & 77\end{array}$

Don't know or can't remember $\quad 4 \quad 0.4$

Nutrition education

No 329

31.9

Yes

691

67

Don't know or can't remember

11

1.1

Breastfeeding education ${ }^{a}$

No 
Table 3 Distribution of quality of antenatal care variables (Continued)

\begin{tabular}{|c|c|c|}
\hline Experience of care & No. & $\%$ \\
\hline Yes & 655 & 63.6 \\
\hline Don't know or can't remember & 10 & 1 \\
\hline \multicolumn{3}{|c|}{ Understood purpose of tests performed } \\
\hline No, Never & 170 & 16.5 \\
\hline Yes, A Few Times & 178 & 17.3 \\
\hline Yes, Most Of The Time & 234 & 22.7 \\
\hline Yes, All The Time & 442 & 42.9 \\
\hline Don't know or can't remember & 7 & 0.7 \\
\hline \multicolumn{3}{|c|}{ Understood purpose of medicines received } \\
\hline No, Never & 154 & 14.9 \\
\hline Yes, A Few Times & 167 & 16.2 \\
\hline Yes, Most Of The Time & 240 & 23.3 \\
\hline Yes, All The Time & 462 & 44.8 \\
\hline Don't know or can't remember & 8 & 0.8 \\
\hline \multicolumn{3}{|l|}{ Felt able to ask any questions ${ }^{a}$} \\
\hline No, Never & 178 & 17.3 \\
\hline Yes, A Few Times & 216 & 21 \\
\hline Yes, Most Of The Time & 195 & 19 \\
\hline Yes, All The Time & 434 & 42.2 \\
\hline Don't know or can't remember & 6 & 0.6 \\
\hline \multicolumn{3}{|l|}{ Asked if she had any questions ${ }^{a}$} \\
\hline No, Never & 306 & 29.7 \\
\hline Yes, A Few Times & 206 & 20 \\
\hline Yes, Most Of The Time & 153 & 14.9 \\
\hline Yes, All The Time & 358 & 34.8 \\
\hline Don't know or can't remember & 7 & 0.7 \\
\hline \multicolumn{3}{|l|}{ Felt treated with respect } \\
\hline No, Never & 15 & 1.5 \\
\hline Yes, A Few Times & 82 & 8 \\
\hline Yes, Most Of The Time & 230 & 22.3 \\
\hline Yes, All The Time & 699 & 67.8 \\
\hline Don't know or can't remember & 5 & 0.5 \\
\hline \multicolumn{3}{|l|}{ Treated in friendly manner ${ }^{\mathrm{a}}$} \\
\hline No, Never & 25 & 2.4 \\
\hline Yes, A Few Times & 109 & 10.6 \\
\hline Yes, Most Of The Time & 247 & 24 \\
\hline Yes, All The Time & 646 & 62.7 \\
\hline Don't know or can't remember & 3 & 0.3 \\
\hline \multicolumn{3}{|l|}{ Could discuss issues in private } \\
\hline No, Never & 316 & 30.6 \\
\hline Yes, A Few Times & 134 & 13 \\
\hline Yes, Most Of The Time & 139 & 13.5 \\
\hline Yes, All The Time & 438 & 42.5 \\
\hline Don't know or can't remember & 4 & 0.4 \\
\hline
\end{tabular}

Table 3 Distribution of quality of antenatal care variables (Continued)

\begin{tabular}{lll}
\hline Experience of care & No. & $\%$ \\
\hline No, Never & 64 & 6.2 \\
Yes, A Few Times & 126 & 12.2 \\
Yes, Most Of The Time & 231 & 22.4 \\
Yes, All The Time & 599 & 58.1 \\
Don't know or can't remember & 11 & 1.1 \\
Asked to give bribes ${ }^{\text {a }}$ & & \\
No, Never & 912 & 88.5 \\
Yes, A Few Times & 64 & 6.2 \\
Yes, Most Of The Time & 29 & 2.8 \\
Yes, All The Time & 24 & 2.3 \\
Don't know or can't remember & 1 & 0.1 \\
Felt treated differently because of any personal attribute & \\
No, Never & 965 & 93.7 \\
Yes, A Few Times & 36 & 3.5 \\
Yes, Most Of The Time & 10 & 1 \\
Yes, All The Time & 16 & 1.6 \\
Don't know or can't remember & 3 & 0.3 \\
Total & 1031 & 100 \\
\hline
\end{tabular}

Notes: All totals equal 1031 except where noted by an ${ }^{\text {a }}$; these have total of $<1031$ due to missing data. The last two items on bribes and differential treatment were not included in the summative score because they loaded poorly on the dominant factor in the factor analysis

Compared to Luo women, Kuria women had higher experience scores and slightly higher service provision scores, but had lower odds of receiving an ultrasound.

Women who were more empowered, from high SES households, had someone in their household working in a health facility, and had health insurance, had on average, higher experience of care scores compared to less empowered women, women from lower SES households, women who had no one in their household working in a health facility, and women who had no health insurance, respectively. The significance and direction of the associations between service provision and the empowerment and SES measures are similar. College educated and women from the wealthiest households have over five times higher odds of receiving an ultrasound than women with less than primary education and those from the poorest households.

Additionally, compared to women who had never experienced domestic violence, women who had experienced domestic violence had lower experience and service provision scores and had lower odds of getting an ultrasound. Also, women who had a severe pregnancy complication and first presented for ANC because of a problem had lower experience scores than those who had no severe pregnancy complication and first presented for ANC for checkup. Women who had any 
Table 4 Bivariate regressions of antenatal care quality measures on various predictors, PQCC 2016/2017

\begin{tabular}{|c|c|c|c|c|c|c|c|c|c|}
\hline \multirow{3}{*}{ Age } & \multicolumn{6}{|c|}{ Linear regression: Coefficient [95\%Cl] } & \multirow{2}{*}{\multicolumn{3}{|c|}{$\frac{\text { Logistic regression: OR }[95 \% \mathrm{Cl}]}{\text { Received an ultrasound }}$}} \\
\hline & \multicolumn{3}{|c|}{ Experience score } & \multicolumn{3}{|c|}{ Service provision score } & & & \\
\hline & & & & & & & & & \\
\hline 15 to 19 years & 0 & {$[0$} & 0] & 0 & {$[0$} & 0] & 1 & {$[1$} & 1] \\
\hline 20 to 29 years & $1.98^{* *}$ & {$[0.55$} & 3.41] & 0.18 & {$[-0.25$} & $0.61]$ & $2.40^{* *}$ & {$[1.34$} & 4.32] \\
\hline 30 to 48 years & $1.80^{*}$ & {$[0.17$} & 3.43] & -0.11 & {$[-0.60$} & $0.38]$ & $2.84^{* *}$ & {$[1.51$} & 5.31] \\
\hline \multicolumn{10}{|l|}{ Marital status } \\
\hline Single & 0 & {$[0$} & 0] & 0 & {$[0$} & 0] & 1 & {$[1$} & 1] \\
\hline Partnered/Cohabiting & -2.88 & {$[-11.0$} & $5.27]$ & -1.59 & {$[-3.98$} & $0.80]$ & 1.38 & {$[0.14$} & 13.7] \\
\hline Married & $2.70^{* * *}$ & {$[1.21$} & 4.19] & -0.17 & {$[-0.61$} & $0.27]$ & 0.77 & {$[0.49$} & 1.20] \\
\hline Widowed & 1.01 & {$[-1.82$} & $3.84]$ & -0.33 & {$[-1.15$} & $0.50]$ & 0.59 & {$[0.23$} & $1.52]$ \\
\hline Divorced/Separated & 2.7 & {$[-2.13$} & 7.54] & 0.18 & {$[-1.30$} & $1.66]$ & 0.75 & {$[0.16$} & 3.57] \\
\hline \multicolumn{10}{|l|}{ Number of births } \\
\hline 1 & 0 & {$[0$} & 0] & 0 & {$[0$} & 0] & 1 & {$[1$} & 1] \\
\hline 2 & 0.05 & {$[-1.46$} & $1.56]$ & -0.085 & {$[-0.52$} & $0.35]$ & 1.4 & {$[0.91$} & 2.14] \\
\hline 3 & 0.16 & {$[-1.38$} & $1.69]$ & $-0.52^{*}$ & {$[-0.97$} & $-0.069]$ & $0.53^{*}$ & {$[0.31$} & $0.91]$ \\
\hline 4 or more & -0.65 & {$[-2.01$} & $0.70]$ & $-1.08^{* * *}$ & {$[-1.47$} & $-0.68]$ & 0.67 & {$[0.43$} & $1.04]$ \\
\hline \multicolumn{10}{|l|}{ Tribe } \\
\hline Luo & 0 & {$[0$} & 0] & 0 & {$[0$} & 0] & 1 & {$[1$} & 1] \\
\hline Kuria & $3.96^{* * *}$ & {$[2.71$} & $5.20]$ & $0.59^{* *}$ & {$[0.22$} & $0.96]$ & $0.28^{* * *}$ & {$[0.16$} & $0.49]$ \\
\hline Other & 1.2 & {$[-0.61$} & $3.01]$ & -0.2 & {$[-0.75$} & $0.35]$ & 1.05 & {$[0.62$} & 1.80] \\
\hline \multicolumn{10}{|l|}{ Religious affiliation } \\
\hline Catholic & 0 & {$[0$} & 0] & 0 & {$[0$} & 0] & 1 & {$[1$} & 1] \\
\hline Protestant/Pentecostal & 0.77 & {$[-0.75$} & $2.28]$ & -0.16 & {$[-0.60$} & $0.28]$ & $0.58^{*}$ & {$[0.36$} & 0.93] \\
\hline Seventh Day Adventist & 0.55 & {$[-0.87$} & 1.98] & -0.11 & {$[-0.52$} & $0.30]$ & 0.99 & {$[0.66$} & 1.48] \\
\hline Other Christian & -0.46 & {$[-1.99$} & $1.06]$ & $-0.58^{*}$ & {$[-1.04$} & $-0.13]$ & $0.31^{* * *}$ & {$[0.17$} & $0.57]$ \\
\hline Muslim/other religion & -2.48 & {$[-6.42$} & $1.45]$ & -1.21 & {$[-2.42$} & $0.0026]$ & 0.2 & {$[0.026$} & 1.51] \\
\hline \multicolumn{10}{|l|}{ Education } \\
\hline No school/Primary & 0 & {$[0$} & 0] & 0 & {$[0$} & 0] & 1 & {$[1$} & 1] \\
\hline Post-primary/vocational/Secondary & $2.15^{* * *}$ & {$[0.96$} & 3.34] & $0.66^{* * *}$ & {$[0.32$} & $1.01]$ & $2.03^{* * *}$ & {$[1.35$} & 3.04] \\
\hline College or above & $1.79^{*}$ & {$[0.11$} & 3.47] & $1.17^{* * *}$ & {$[0.69$} & $1.66]$ & $9.22^{* * *}$ & {$[5.87$} & 14.5] \\
\hline Literate & $2.28^{* * *}$ & {$[1.05$} & $3.51]$ & $0.72^{* * *}$ & {$[0.35$} & $1.08]$ & $2.57^{* * *}$ & {$[1.57$} & 4.19] \\
\hline Employed & $3.66^{* * *}$ & {$[2.44$} & 4.88] & $0.86^{* * *}$ & {$[0.50$} & $1.22]$ & $2.14^{* * *}$ & {$[1.50$} & 3.06] \\
\hline \multicolumn{10}{|l|}{ Household wealth quintile } \\
\hline Poorest/Poorer & 0 & {$[0$} & 0] & 0 & {$[0$} & 0] & 1 & {$[1$} & 1] \\
\hline Middle & 0.73 & {$[-0.80$} & $2.26]$ & 0.45 & {$[-0.000034$} & $0.90]$ & 1.67 & {$[0.93$} & $3.01]$ \\
\hline Richer/Richest & $1.56^{* *}$ & {$[0.40$} & $2.72]$ & $0.78^{* * *}$ & {$[0.44$} & 1.12] & $5.03^{* * *}$ & {$[3.35$} & 7.53] \\
\hline \multicolumn{10}{|l|}{ Current occupation } \\
\hline Agricultural labor & 0 & {$[0$} & 0] & 0 & {$[0$} & 0] & 1 & {$[1$} & 1] \\
\hline Casual labor & -0.71 & {$[-3.18$} & 1.77] & 0.45 & {$[-0.29$} & 1.19] & 1.86 & {$[0.76$} & 4.53] \\
\hline Salaried worker & $3.16^{* *}$ & {$[1.02$} & $5.30]$ & $0.79^{*}$ & {$[0.17$} & 1.42] & $8.51^{* * *}$ & {$[4.32$} & 16.8] \\
\hline Self-employed in petty trade & $3.07^{* * *}$ & {$[1.28$} & 4.85] & 0.53 & {$[-0.00080$} & $1.05]$ & $2.10^{*}$ & {$[1.07$} & 4.12] \\
\hline Self-employed small-scale industry & 1.18 & {$[-2.17$} & 4.53] & 0.28 & {$[-0.72$} & $1.28]$ & $3.55^{*}$ & {$[1.29$} & 9.75] \\
\hline Unemployed/homemaker & 0.58 & {$[-0.94$} & $2.10]$ & 0.19 & {$[-0.26$} & $0.64]$ & 1.7 & {$[0.93$} & 3.13] \\
\hline Other & 1.31 & {$[-3.92$} & $6.54]$ & -0.017 & {$[-1.72$} & 1.69] & 3.34 & {$[0.82$} & 13.6] \\
\hline
\end{tabular}


Table 4 Bivariate regressions of antenatal care quality measures on various predictors, PQCC 2016/2017 (Continued)

\begin{tabular}{|c|c|c|c|c|c|c|c|c|c|}
\hline & \multicolumn{6}{|c|}{ Linear regression: Coefficient [95\%Cl] } & \multirow{2}{*}{\multicolumn{3}{|c|}{$\frac{\text { Logistic regression: OR }[95 \% \mathrm{Cl}]}{\text { Received an ultrasound }}$}} \\
\hline & \multicolumn{3}{|c|}{ Experience score } & \multicolumn{3}{|c|}{ Service provision score } & & & \\
\hline & \multirow[b]{2}{*}{0} & \multirow[b]{2}{*}{0} & \multirow[b]{2}{*}{ 0] } & \multirow[b]{2}{*}{0} & \multirow[b]{2}{*}{0} & \multirow[b]{2}{*}{ 0] } & \multirow[b]{2}{*}{1} & & \\
\hline Partner's education & & & & & & & & {$[1$} & 1] \\
\hline Post-primary/vocational/Secondary & 1.1 & {$[-0.26$} & $2.46]$ & 0.3 & {$[-0.093$} & $0.70]$ & $1.74^{*}$ & {$[1.05$} & 2.89] \\
\hline College or above & $2.22^{* *}$ & {$[0.64$} & 3.81] & $1.25^{* * *}$ & {$[0.79$} & $1.72]$ & $7.59^{* * *}$ & {$[4.67$} & 12.3] \\
\hline No Partner & $-1.54^{*}$ & {$[-2.96$} & $-0.12]$ & $0.44^{*}$ & {$[0.025$} & $0.85]$ & $2.36^{* * *}$ & {$[1.43$} & 3.89] \\
\hline \multicolumn{10}{|l|}{ Partner's occupation } \\
\hline Agricultural labor & 0 & {$[0$} & 0] & 0 & {$[0$} & 0] & 1 & {$[1$} & 1] \\
\hline Casual labor & -1.68 & {$[-3.36$} & $0.0057]$ & 0.15 & {$[-0.36$} & $0.65]$ & 1.32 & {$[0.68$} & 2.53] \\
\hline Salaried worker & 1.29 & {$[-0.45$} & 3.03] & $0.91^{* * *}$ & {$[0.38$} & 1.43] & $4.77^{* * *}$ & {$[2.68$} & $8.51]$ \\
\hline Self-employed in petty trade & 1.69 & {$[-0.11$} & 3.49] & 0.51 & {$[-0.029$} & 1.04] & 1.01 & {$[0.48$} & 2.12] \\
\hline Self-employed small-scale industry & -0.74 & {$[-2.90$} & 1.41] & 0.6 & {$[-0.041$} & 1.24] & $2.19^{*}$ & {$[1.05$} & $4.54]$ \\
\hline Unemployed/homemaker & -0.62 & {$[-4.21$} & 2.98] & 0.17 & {$[-0.91$} & $1.25]$ & $7.29^{* * *}$ & {$[2.85$} & 18.6] \\
\hline Other & -4.75 & {$[-12.8$} & 3.31] & 0.45 & {$[-1.92$} & 2.83] & 1 & {$[1$} & 1] \\
\hline No Partner & $-2.23^{* *}$ & {$[-3.86$} & $-0.60]$ & $0.48^{*}$ & {$[0.0028$} & $0.97]$ & $2.19^{* *}$ & {$[1.22$} & 3.94] \\
\hline Self or family work in health facility & $2.95^{* *}$ & {$[0.74$} & $5.17]$ & $0.72^{*}$ & {$[0.073$} & $1.36]$ & $1.97^{*}$ & {$[1.10$} & 3.51] \\
\hline Has health insurance & $3.08^{* * *}$ & {$[1.66$} & 4.50] & $0.86^{* * *}$ & {$[0.44$} & 1.29] & $3.45^{* * *}$ & {$[2.36$} & $5.05]$ \\
\hline High participation in household decisions & $1.30^{*}$ & {$[0.25$} & 2.35] & -0.011 & {$[-0.32$} & $0.30]$ & $2.68^{* * *}$ & {$[1.88$} & 3.81] \\
\hline Intolerant towards domestic violence & $1.59^{* *}$ & {$[0.54$} & 2.64] & $0.86^{* * *}$ & {$[0.55$} & 1.17] & $2.89^{* * *}$ & {$[2.00$} & 4.18] \\
\hline Experienced domestic violence & $-2.48^{* * *}$ & {$[-3.53$} & $-1.44]$ & $-1.12^{* * *}$ & {$[-1.42$} & $-0.82]$ & $0.55^{* * *}$ & {$[0.39$} & $0.77]$ \\
\hline Had any pregnancy complications & 0.84 & {$[-0.22$} & 1.90] & $-0.40^{*}$ & {$[-0.71$} & $-0.092]$ & $0.65^{*}$ & {$[0.46$} & $0.92]$ \\
\hline Had severe pregnancy complications & $1.45^{*}$ & {$[0.31$} & 2.59] & -0.27 & {$[-0.61$} & $0.061]$ & 0.73 & {$[0.50$} & 1.06] \\
\hline Had complications in prior pregnancy & -0.53 & {$[-2.08$} & 1.03] & $-0.51^{*}$ & {$[-0.98$} & $-0.047]$ & 0.93 & {$[0.56$} & 1.52] \\
\hline Received ANC in prior pregnancy & -0.011 & {$[-1.13$} & $1.11]$ & $-0.51^{* *}$ & {$[-0.84$} & $-0.19]$ & 0.9 & {$[0.64$} & 1.28] \\
\hline Prior facility delivery & 0.63 & {$[-0.45$} & $1.71]$ & -0.25 & {$[-0.57$} & $0.073]$ & 1.04 & {$[0.74$} & 1.47] \\
\hline \multicolumn{10}{|l|}{ Reason for first ANC } \\
\hline Because of a problem & 0 & {$[0$} & 0] & 0 & {$[0$} & 0] & 1 & {$[1$} & 1] \\
\hline Just for a checkup & $1.69^{*}$ & {$[0.033$} & 3.34] & 0.077 & {$[-0.43$} & $0.59]$ & 0.77 & {$[0.47$} & $1.27]$ \\
\hline Can't Remember & -4.45 & {$[-10.0$} & $1.13]$ & 0.25 & {$[-1.48$} & 1.99] & 0.51 & {$[0.061$} & 4.31] \\
\hline \multicolumn{10}{|l|}{ Timing of first antenatal visit } \\
\hline First trimester & 0 & {$[0$} & 0] & 0 & {$[0$} & 0] & 1 & {$[1$} & 1] \\
\hline Second trimester & -0.48 & {$[-1.66$} & $0.70]$ & -0.032 & {$[-0.38$} & $0.31]$ & 0.87 & {$[0.60$} & $1.25]$ \\
\hline Third Trimester & $-3.31^{* *}$ & {$[-5.31$} & $-1.31]$ & $-1.36^{* * *}$ & {$[-1.94$} & $-0.78]$ & 0.64 & {$[0.33$} & $1.26]$ \\
\hline \multicolumn{10}{|l|}{ Number of antenatal visits } \\
\hline Less than 4 & 0 & {$[0$} & 0] & 0 & {$[0$} & 0] & 1 & {$[1$} & 1] \\
\hline 4 or 5 & $1.50^{* *}$ & {$[0.37$} & 2.64] & $0.70^{* * *}$ & {$[0.36$} & $1.03]$ & $1.57^{*}$ & {$[1.07$} & 2.29] \\
\hline 6 plus & $2.34^{*}$ & {$[0.53$} & $4.15]$ & $1.24^{* * *}$ & {$[0.72$} & $1.77]$ & 1.75 & {$[1.00$} & 3.06] \\
\hline \multicolumn{10}{|l|}{ Highest ANC facility } \\
\hline Gov't Hospital & 0 & {$[0$} & 0] & 0 & {$[0$} & 0] & 1 & {$[1$} & 1] \\
\hline Gov't HC/Dispensary & 0.68 & {$[-0.46$} & $1.81]$ & $-0.52^{* *}$ & {$[-0.86$} & $-0.19]$ & $0.28^{* * *}$ & {$[0.19$} & $0.41]$ \\
\hline Mission/Private facility & $3.48^{* * *}$ & {$[1.60$} & $5.36]$ & 0.5 & {$[-0.045$} & $1.05]$ & $1.62^{*}$ & {$[1.01$} & 2.59] \\
\hline Highest ANC Provider type & & & & & & & & & \\
\hline Nurse/Midwife & 0 & {$[0$} & 0] & 0 & {$[0$} & 0] & 1 & {$[1$} & 1] \\
\hline Doctor/Clinical officer & 1.37 & {$[-0.27$} & 3.02] & -0.16 & {$[-0.66$} & $0.34]$ & 0.69 & {$[0.38$} & 1.24] \\
\hline
\end{tabular}


Table 4 Bivariate regressions of antenatal care quality measures on various predictors, PQCC 2016/2017 (Continued)

\begin{tabular}{|c|c|c|c|c|c|c|c|c|c|}
\hline \multirow[b]{3}{*}{ Non-skilled attendant } & \multicolumn{6}{|c|}{ Linear regression: Coefficient $[95 \% \mathrm{Cl}]$} & \multirow{2}{*}{\multicolumn{3}{|c|}{$\frac{\text { Logistic regression: OR }[95 \% \mathrm{CI}]}{\text { Received an ultrasound }}$}} \\
\hline & \multicolumn{3}{|c|}{ Experience score } & \multicolumn{3}{|c|}{ Service provision score } & & & \\
\hline & 2.75 & {$[-2.66$} & $8.15]$ & -0.051 & {$[-1.48$} & 1.38] & 1.11 & {$[0.24$} & $5.17]$ \\
\hline In the community/a home & $-2.19 * * *$ & {$[-3.25$} & $-1.13]$ & $-0.58^{* * *}$ & {$[-0.90$} & $-0.26]$ & 0.92 & {$[0.65$} & $1.28]$ \\
\hline Postpartum length $>=1$ week & $-2.97^{* *}$ & {$[-4.92$} & $-1.03]$ & $-0.88^{* *}$ & {$[-1.45$} & $-0.31]$ & 1.12 & {$[0.59$} & 2.12] \\
\hline
\end{tabular}

Notes: $95 \%$ confidence intervals in brackets ${ }^{*} p<0.05{ }^{* *} p<0.01{ }^{* * *} p<0.001$

complication, however, had lower service provision scores, with lower odds of receiving an ultrasound.

Women who started ANC in the first trimester, received ANC four or more times, and solely from private facilities have higher experience scores than those who started ANC after the first trimester, received ANC less than four times, and from government facilities, respectively. Similarly, women who started ANC in the first trimester and who received ANC four or more times had higher service provision scores. Service provision scores, however, did not differ between government hospitals and private facilities, but were lower for health centers. Additionally, compared to women who received ANC services in hospitals, women who received ANC in health centers had lower odds of receiving an ultrasound, while those who received ANC solely in a private facility had higher odds of receiving an ultrasound.

\section{Multivariate}

The multivariate models presented in Table 5 shows that, after controlling for other factors the following associations had $p$-values less than 0.05 . On average, women in the 20 to 29 age group still have higher experience scores and those older than 30 years have higher service provision scores than those younger than 20 years. Both age groups are also over two times more likely to have done an ultrasound test than the younger women. Women with four or more children have lower service provision scores than the primiparous women. Net of other factors, Kuria women still had higher experience scores and slightly higher service provision scores, but had lower odds of receiving an ultrasound than Luo women.

After controlling for other factors, women who are literate, employed, and participate in household decisions also still have higher experiences scores, but only employment is significant for higher service provision, and those with higher participation in household decision making are more likely to get an ultrasound. Household wealth and partner's education are significantly associated with getting an ultrasound, with women from the wealthiest households and those with college educated husbands having about two times higher odds of receiving an ultrasound than women from the poorest households and whose husbands have less than primary education. Controlling for other factors, women who had experienced domestic violence still had lower experience and service provision scores than women who had never experienced domestic violence.

Women who started ANC in the third trimester had lower experience and service provision scores, and those who received ANC four or more times received slightly higher service provision scores. Timing and frequency of ANC is not significantly associated with odds of getting an ultrasound. Additionally, compared to women who received ANC in hospitals, women who received ANC in health centers had higher experience scores but lower odds of receiving an ultrasound, while those who received ANC solely in a private facility had higher experience scores, but no difference in service provision scores or the odds of getting an ultrasound. The effect of location of the interview persists after controlling for other factors.

\section{Discussion}

This is one of the few studies to examine both service provision and experience dimensions of quality of ANC in a low resource setting, and to our knowledge, the first to do this at sub-national level in Kenya. We find that ANC quality is suboptimal in terms of providing recommended ANC services as well as ensuring women have a good experience. While many women receive basic ANC services such as blood pressure monitoring and urine test at least once during pregnancy, many are not receiving these consistently at every visit as recommended by the Kenya National guidelines [13]. The situation is even more dire for more advanced services such as ultrasounds, which less than one out of every five women in our sample received, with women who had complications (the group for whom it is recommended) less likely to receive it.

Although there is increased attention to mistreatment and poor person-centered care in facilities globally, most of this work has focused on intrapartum care. In this paper we also draw attention to poor PCANC, which can affect women's adherence to treatment recommendations and deter them from returning to a facility to give birth [37-39]. The major gap in PCANC, which has been shown in other work for maternity care, is in the domain of communication [40]: women are not given sufficient information during ANC about their care, hence do not understand the purpose of examinations 
Table 5 Multivariate regressions of antenatal care quality measures on various predictors, PQCC 2016/2017

\begin{tabular}{|c|c|c|c|c|c|c|c|c|c|}
\hline & \multicolumn{6}{|c|}{ Linear regression: Coefficient [95\% Cl] } & \multirow{2}{*}{\multicolumn{3}{|c|}{$\begin{array}{l}\text { Logistic regression: OR [95\% CI] } \\
\text { Received an ultrasound }\end{array}$}} \\
\hline & \multicolumn{3}{|c|}{ Experience score } & \multicolumn{3}{|c|}{ Service provision score } & & & \\
\hline & & & & & & & & & \\
\hline 15 to 19 years & 0 & {$[0$} & 0] & 0 & {$[0$} & 0] & 1 & {$[1$} & 1] \\
\hline 20 to 29 years & $1.72^{*}$ & {$[0.088$} & 3.35] & 0.47 & {$[-0.0069$} & $0.96]$ & $2.17^{*}$ & {$[1.06$} & 4.45] \\
\hline 30 to 48 years & 1.78 & {$[-0.35$} & $3.91]$ & $0.67^{*}$ & {$[0.034$} & 1.30] & $2.92^{*}$ & {$[1.21$} & 7.04] \\
\hline Currently married & 2.94 & {$[-4.57$} & $10.5]$ & 1.39 & {$[-0.81$} & 3.59] & 0.55 & {$[0.043$} & 6.91] \\
\hline \multicolumn{10}{|l|}{ Number of births } \\
\hline 1 & 0 & {$[0$} & 0] & 0 & {$[0$} & 0] & 1 & {$[1$} & 1] \\
\hline 2 & -0.84 & {$[-2.45$} & $0.77]$ & -0.015 & {$[-0.49$} & $0.46]$ & 1.26 & {$[0.72$} & 2.22] \\
\hline 3 & -0.67 & {$[-2.41$} & $1.08]$ & -0.36 & {$[-0.88$} & $0.16]$ & 0.52 & {$[0.26$} & 1.03] \\
\hline 4 or more & -0.88 & {$[-2.74$} & $0.99]$ & $-0.71^{*}$ & {$[-1.26$} & $-0.16]$ & 0.86 & {$[0.43$} & 1.72] \\
\hline \multicolumn{10}{|l|}{ Tribe } \\
\hline Luo & 0 & {$[0$} & 0] & 0 & {$[0$} & 0] & 1 & {$[1$} & 1] \\
\hline Kuria & $4.97^{* * *}$ & {$[3.70$} & $6.25]$ & $0.89^{* * *}$ & {$[0.52$} & $1.27]$ & $0.37^{* *}$ & {$[0.20$} & $0.69]$ \\
\hline Other & 1.57 & {$[-0.16$} & $3.31]$ & -0.068 & {$[-0.59$} & $0.45]$ & 1.3 & {$[0.70$} & 2.41] \\
\hline Literate & $1.52^{*}$ & {$[0.26$} & $2.79]$ & 0.22 & {$[-0.16$} & $0.60]$ & 1.35 & {$[0.76$} & 2.38] \\
\hline Employed & $2.73^{* * *}$ & {$[1.46$} & $4.00]$ & $0.56^{* *}$ & {$[0.19$} & $0.93]$ & 1.01 & {$[0.64$} & 1.59] \\
\hline Participation in household decisions & $1.24^{*}$ & {$[0.14$} & $2.34]$ & -0.16 & {$[-0.49$} & $0.17]$ & $1.77^{*}$ & {$[1.14$} & 2.75] \\
\hline \multicolumn{10}{|l|}{ Household wealth } \\
\hline Poorest/poorer & 0 & {$[0$} & 0] & 0 & {$[0$} & 0] & 1 & {$[1$} & 1] \\
\hline Middle & 0.99 & {$[-0.49$} & 2.47] & 0.27 & {$[-0.17$} & $0.71]$ & 1.03 & {$[0.53$} & 2.02] \\
\hline Richer/richest & 0.7 & {$[-0.64$} & 2.04] & 0.13 & {$[-0.27$} & $0.53]$ & $2.00^{* *}$ & {$[1.20$} & 3.33] \\
\hline \multicolumn{10}{|l|}{ Partner's education } \\
\hline No school/Primary & 0 & {$[0$} & 0] & 0 & {$[0$} & 0] & 1 & {$[1$} & 1] \\
\hline Post-primary/vocational/Secondary & 0.16 & {$[-1.21$} & 1.52] & -0.062 & {$[-0.47$} & $0.35]$ & 0.92 & {$[0.52$} & 1.64] \\
\hline College or above & -0.46 & {$[-2.27$} & $1.36]$ & 0.39 & {$[-0.15$} & $0.93]$ & $2.40^{* *}$ & {$[1.29$} & 4.46] \\
\hline No Partner & 1.42 & {$[-6.20$} & $9.03]$ & 1.53 & {$[-0.71$} & $3.76]$ & 0.94 & {$[0.072$} & 12.4] \\
\hline Experienced domestic violence & $-2.42^{* * *}$ & {$[-3.51$} & $-1.33]$ & $-0.83^{* * *}$ & {$[-1.15$} & $-0.51]$ & 0.91 & {$[0.60$} & 1.36] \\
\hline Had severe complications & 0.91 & {$[-0.19$} & 2.02] & -0.24 & {$[-0.56$} & $0.088]$ & 1.19 & {$[0.77$} & 1.85] \\
\hline \multicolumn{10}{|l|}{ Timing of first antenatal visit } \\
\hline First trimester & 0 & {$[0$} & 0] & 0 & {$[0$} & 0] & 1 & {$[1$} & 1] \\
\hline Second trimester & -0.17 & {$[-1.32$} & $0.98]$ & 0.16 & {$[-0.19$} & $0.50]$ & 1.18 & {$[0.76$} & 1.81] \\
\hline Third Trimester & $-2.21^{*}$ & {$[-4.28$} & $-0.15]$ & $-0.75^{*}$ & {$[-1.36$} & $-0.13]$ & 0.82 & {$[0.35$} & 1.93] \\
\hline Four plus antenatal visits & 0.34 & {$[-0.81$} & 1.49] & $0.38^{*}$ & {$[0.033$} & $0.72]$ & 1.1 & {$[0.70$} & 1.74] \\
\hline \multicolumn{10}{|l|}{ Highest ANC facility } \\
\hline Gov't Hospital & 0 & {$[0$} & 0] & 0 & {$[0$} & 0] & 1 & {$[1$} & 1] \\
\hline Gov't HC/Dispensary & $1.99^{* * *}$ & {$[0.84$} & 3.14] & -0.085 & {$[-0.43$} & $0.25]$ & $0.33^{* * *}$ & {$[0.21$} & $0.52]$ \\
\hline Mission/Private facility & $3.28^{* * *}$ & {$[1.48$} & 5.09] & 0.48 & {$[-0.050$} & $1.01]$ & 1.31 & {$[0.76$} & 2.24] \\
\hline Interviews in the community & $-2.41^{* * *}$ & {$[-3.44$} & $-1.38]$ & $-0.55^{* * *}$ & {$[-0.86$} & $-0.24]$ & 1.08 & {$[0.73$} & 1.61] \\
\hline Constant & $20.9^{* * *}$ & {$[13.1$} & 28.6] & $9.38^{* * *}$ & {$[7.11$} & 11.7] & 0.08 & {$[0.0056$} & 1.16] \\
\hline N & 909 & & & 882 & & & 993 & & \\
\hline R-squared & 0.179 & & & 0.172 & & & & & \\
\hline
\end{tabular}


and medicines, but are not able to ask clarifying questions. Most women felt respected by their health care providers, which is encouraging. However, that 1 in 10 women did not feel respected means there is room for improvement, given the internalization and normalization of disrespect which usually results in low reporting when compared to observations [41-43]. We did not include survey questions on extreme forms of poor person-centered care such as verbal and physical abuse. But prior qualitative work in this setting suggests that verbal and physical abuse sometimes occurs during ANC [44].

As in many areas of health care, the most disadvantaged and disempowered women receive the lowest quality ANC relating to both service provision and experience of care. The potential reasons why more empowered and wealthier women are more likely to receive high quality ANC and person-centered care is described in detail elsewhere $[20,25,40]$. These reasons include being able to access care in facilities that offer higher quality care, being able to pay for higher quality care, and having the knowledge and ability to advocate for higher quality care. Studies in Kenya have shown that quality of care is poorer in low resource communities where poor women tend to live [22]. In this paper, we also find that the SES and empowerment differences are more marked for the experience of care dimensions than for the provision of ANC services. This is potentially because the services included in the service provision index are basic services that are offered free of charge to most clients, thus requiring less knowledge or ability to advocate for them.

The exception is in getting an ultrasound where SES measured by household wealth and partner's education is a significant predictor. This finding is not surprising given the limited availability of ultrasounds in many government facilities in this setting. At the time of this survey, even the referral hospital had no functional ultrasound. Ultrasounds are also not covered by the National Health Insurance funds. This required that women who needed ultrasounds obtain it at private facilities where they had to pay before getting the services. Ultrasound costs in private facilities in Kenya vary widely (between 600 and 4000 Kenyan shillings (about 6 to 40 dollars) because of lack of regulation [45]. Furthermore, women with complications for whom ultrasound is recommended were less likely to receive an ultrasound than those with no complications. Therefore, those who need it the most may not be getting it because of cost due to systemic weaknesses. The higher odds of receiving an ultrasound among women who received some ANC in a hospital is likely because women receiving ANC in a hospital may have been referred there because of a complication, prompting providers there to request an ultrasound test. Ethnic differences between Luo and Kuria women in ANC quality might be due to biases against or in favor of certain ethnicities resulting in them receiving less services and being treated differently. We believe implicit bias plays an important role in quality of care differentials in this setting not just by ethnicity, but also based on SES and age, and thus account for some of those disparities too. Policies and interventions to improve quality of care therefore need consider how to address factors that contribute to these disparities.

The findings also suggest that certain high-risk women may not be getting key recommended services. For example, younger women (15 to 19 years) are less likely to get an ultrasound, in addition to being less likely have good PCANC. Given that this age group have high risk of complications, poor quality care may be playing a big role in their outcomes as complications may not be identified early or at all. In addition, perceptions of poor person-centered care may deter them from starting ANC early and attending frequently, further delaying identification of complications, and they may be less likely to deliver in a health facility where complications can be managed. Poor ANC quality in this group thus has detrimental consequences. Another high-risk group that was less likely to consistently receive the basic antenatal service was women with 4 or more children. This might be due to less attention to these women because of their prior childbirth experience, which could lead to adverse consequences for them if they receive less screening and preventative services. Other factors that account for differences in ANC quality are the timing and frequency of ANC. Both timing and frequency of ANC are important for the number of services one receives, but not for whether or not a woman gets an ultrasound. However, only timing is associated with experience of care, with women who received ANC in the third trimester reporting poorer experiences. This might be due to insufficient time for counselling and mistreatment from providers when women present for ANC late in the pregnancy, which we found in our qualitative work [44].

In addition, the types of facility where one receives care affects the quality of care they receive based on different dimensions. In general, there was no difference in service provision scores by facility. However, women who received ANC at least once from a government hospital had lower experience scores, but had higher odds of getting an ultrasound. On the other hand, those who received care in a health center, had higher experience scores, but had lower odds of getting an ultrasound. Women who received care in only a private facility also had higher experience scores, but had similar odds of getting an ultrasound as those who were seen in the government hospital. The finding of higher experience scores in health centers and private facilities is consistent with prior studies on women's experiences for antenatal and delivery 
care and for family planning services [20, 40, 46, 47]. However, it raises the question of where the 'best' care for women might be during ANC [25]. Most women, particularly poor women, do not have the option of receiving care in private facilities. While they may receive more advanced essential services in the higher-level facilities, which have more staffing and clinical infrastructure, they also stand the risk of being mistreated in these facilities. Women should not have to choose between receipt of essential services and good person-centered care. Thus, there is a need for targeted PCANC interventions in higher-level facilities, as well as equipping the lower level facilities to be able to provide the essential antenatal services.

Various reasons, ranging from structural factors to provider attitudes, account for the suboptimal ANC quality. Providers will be unable to take weight and blood pressure measurements or to do blood and urine tests if they do not have working scales and blood pressure monitors or functional laboratories, reagents and supplies needed for these tests. Similarly, they will be unable to give medications they do not have in stock. Thus, availability of necessary equipment, supplies, and medicines are key to providing good quality ANC. These reasons are much more relevant for service provision than experience of care, although the frustration and stress of providing care without all the necessary tools could also manifest in providers' interactions with women. Lack of provider knowledge of service provision guidelines and their knowledge and willingness to provide person-centered care is also a potential reason for poor quality. It is notable in the distribution of the individual measures shown in Table 2 that women were far more likely to be given various services than to be given information and listened to. One reason is that in ANC clinics where one provider may be trying to attend to several women, it is faster to do tests and dispense medication than spending time explaining to women and answering their questions. Thus, poor communication may be because of time constraints or workflow. The implication of this is that women might not be adhering to treatments and recommendations for further tests because they do not understand why these are necessary. Providers therefore need to be able to prioritize effective communication even in busy health facilities.

\section{Limitations}

This study has potential limitations. Firstly, the measures of ANC quality are based on self-report. Recall bias is thus a potential limitation as women may not accurately remember whether or not they received a service. Second, although we assessed the appropriateness of combining the various items to generate the service provision and experience of care scores, the items are not from validated scales. Thus, there is a need for a more systematic process to developing validated scales for ANC service provision and experience of care. Additionally, in creating the summative scores, we coded 'don't know'/ 'don't remember' responses as missing. But it is likely that women who said they can't remember did not receive it, and if they don't know, they likely weren't told about it. Thus, we may have excluded women who received the poorest quality ANC, thus overestimating the actual levels of ANC quality. Social desirability bias is also potentially a limitation if women responded in a way that will please providers. This is likely a problem among women who were interviewed in a health facility and closer to the time of birth, as shown by the higher service provision and experience scores for women who were interviewed in a health facility and within a week of birth compared to women who were interviewed at home and after a week of birth. These are consistent with other findings on women's experiences during childbirth [25, 26, 48]. Furthermore, we used proxy measures of empowerment, which may only partially address cognitive and psychological empowerment, and we are unable to account for structural factors that affect quality of care. Finally, the results are not generalizable to all of Kenya, as data was collected in a specific county using a multistage approach which included convenience samples within randomly selected health units.

Despite these limitations, this study makes valuable contributions to existing research on ANC quality in Kenya and other low-resource settings. It is among the few studies to examine both service provision and experience dimensions of quality of ANC in a low resource setting, thus extending the evidence base for calls to improve quality of ANC and person-centered care. Measuring the different dimensions of quality of care with several items enabled us to identify key areas that need to be addressed to improve quality of care. In addition, the use of composite measures enabled us to include multiple aspects of care provision and experience to assess quality of ANC on a continuum. Moreover, the inclusion of women who received care from different types of facilities enabled us to highlight issues that need to be considered at the different levels and types of facilities.

\section{Conclusions}

This study adds to growing evidence on poor quality of maternal health care. We find that quality of ANC is suboptimal in both domains of service provision and experience of care, with disparities by demographic and socioeconomic factors as well as facility type. Much work is thus needed to improve both dimensions of quality of ANC at different levels of health facilities. In addition, disparities in quality of ANC based on demographic and social status need to be addressed in order to achieve the "no woman left behind" sustainable development goal. While it is still important to 
get women to health facilities, much more is needed to achieve the benefits of ANC, by ensuring that women consistently receive services required to prevent, identify, and manage complications. Furthermore, momentum for improving person-centered maternity care through the respectful maternity care movement should spread to ANC to ensure women are receiving person-centered care along the pregnancy childbirth continuum. As countries such as Kenya update their national guidelines for maternity care to align with new WHO standards, they must consider how to strengthen providers to provide person-centered care to all women in all types of facilities. Further research on the barriers and facilitators to providing high quality ANC will help guide further recommendations to improve quality of ANC and reduce disparities.

\section{Additional file}

Additional file 1: Antenatal care questions from Perceived Quality of Care during Childbirth Study. (PDF 92 kb)

\section{Abbreviations}

ANC: Antenatal care; PCANC: Person-centered antenatal care; WHO: World Health Organization

\section{Acknowledgments}

We are grateful to the PTBI fellowship leaders and the PTBI-East Africa and PTBI-Kenya teams for supporting the study. We thank the leadership of Migori County and the various sub-counties and health facilities, and the community health extension workers and community health volunteers, who facilitated our entry into the County, facilities, and communities. We will like to acknowledge Linet Ouma and Beryl Akinyi Ogola, the PQCC study coordinator and research assistant who coordinated the data collection. Finally, we like to express our gratitude to all our data collectors and study participants in Kenya.

\section{Authors' contributions}

PA led the design and implementation of the study, conceived the manuscript, conducted the analysis, and led the writing. LB supported literature review and writing of the manuscript. FE supported analysis and writing. JK supported implementation and writing. LK and CC served as mentors to PA and guided her in the implementation of the study and supported the writing of the manuscript. All authors have read and approved the manuscript.

\section{Funding}

PA was supported by the University of California, San Francisco, Preterm Birth Initiative (PTBi) transdisciplinary post-doctoral fellowship during the period of this work. This work was part of the Perceived Quality of Care during Childbirth (PQCC) Study nested within the Kenya arm of the PTBi Program. The PQCC study is funded by the PTBi fellowship, which is in turn funded by the Bill and Melinda Gates Foundation [OPP1107312]; and Marc and Lynne Benioff. The funders had no role in the study design, data collection and analysis, decision to publish, or preparation of the manuscript.

\section{Availability of data and materials}

The data analyzed for this manuscript are available from the corresponding author on reasonable request.

\section{Ethics approval and consent to participate}

The study was reviewed and approved by the University of California, San Francisco Committee for Human Subjects research (IRB number 15-18458) and the Kenya Medical Research Institute Scientific and Ethics Review Unit (protocol ID KEMRI/SERU/CCR/039/3286). The Migori County health leadership also approved the study to be conducted in the county. All participants provided written informed consent prior to participation. Respondents 15 to 17 years were considered emancipated minors as they had all given birth. They therefore consented for themselves.

\section{Consent for publication}

Not applicable

\section{Competing interests}

The authors declare that they have no competing interests.

\section{Author details}

${ }^{1}$ School of Medicine, University of California, San Francisco, USA. ${ }^{2}$ Institute for Global Health Sciences, UCSF, San Francisco, USA. '3niversity of New England, Portland, USA. ${ }^{4}$ Kenya Medical Research Institute, Nairobi, Kenya.

Received: 26 October 2018 Accepted: 28 August 2019

Published online: 07 October 2019

\section{References}

1. Alkema L, Chou D, Hogan D, Zhang S, Moller A-B, Gemmill A, et al. Global, regional, and national levels and trends in maternal mortality between 1990 and 2015, with scenario-based projections to 2030: a systematic analysis by the UN maternal mortality estimation inter-agency group. Lancet. 2016;387: 462-74.

2. GBD 2015 Child Mortality Collaborators. Global, regional, national, and selected subnational levels of stillbirths, neonatal, infant, and under-5 mortality, 1980-2015: a systematic analysis for the Global Burden of Disease Study 2015. Lancet Lond Engl. 2016;388:1725-74.

3. Blencowe H, Cousens S, Jassir FB, Say L, Chou D, Mathers C, et al. National, regional, and worldwide estimates of stillbirth rates in 2015, with trends from 2000: a systematic analysis. Lancet Glob Health. 2016:4:e98-108.

4. $\mathrm{WHO} \mid \mathrm{WHO}$ statement on antenatal care. WHO. http://www.who.int/ maternal_child_adolescent/documents/rhr_11_12/en/. Accessed 9 Sep 2013.

5. Chukwuma A, Wosu AC, Mbachu C, Weze K. Quality of antenatal care predicts retention in skilled birth attendance: a multilevel analysis of 28 African countries. BMC Pregnancy Childbirth. 2017;17:152.

6. Adjiwanou V, LeGrand T. Does antenatal care matter in the use of skilled birth attendance in rural Africa: a multi-country analysis. Soc Sci Med. 2013;86:26-34.

7. Afulani PA, Moyer C. Explaining disparities in use of skilled birth attendants in developing countries: a conceptual framework. PLoS One. 2016;11:e0154110.

8. World Health Organisation. WHO recommendations on antenatal care for a positive pregnancy experience. 2016. http://www.who.int/ reproductivehealth/publications/maternal perinatal health/anc-positivepregnancy-experience/en/. Accessed 18 Sep 2018

9. World Health Organization. WHO recommendations: intrapartum care for a positive childbirth experience. WHO. 2018. http//www.who.int/reproductivehealth/ publications/intrapartum-care-guidelines/en/. Accessed 28 Feb 2018.

10. Tunçalp Ö, Were W, MacLennan C, Oladapo O, Gülmezoglu A, Bahl R, et al. Quality of care for pregnant women and newborns-the WHO vision. BJOG Int J Obstet Gynaecol. 2015;122:1045-9.

11. Vogel JP, Habib NA, Souza JP, Gülmezoglu AM, Dowswell T, Carroli G, et al. Antenatal care packages with reduced visits and perinatal mortality: a secondary analysis of the WHO antenatal care trial. Reprod Health. 2013;10:19.

12. $\mathrm{WHO} \mid \mathrm{WHO}$ antenatal care randomized trial. Manual for the implementation of the new model. WHO. http://www.who.int/ reproductivehealth/publications/maternal_perinatal_health/RHR_01_30/en/. Accessed 9 Sep 2013.

13. Ministry of Public Health and Sanitation and Ministry of Medical Services. National Guidelines for Quality Obstetrics and Perinatal Care, Kenya. 2012. https://www.k4health.org/toolkits/kenya-health/national-guidelines-qualityobstetrics-and-perinatal-care. Accessed 26 Aug 2017.

14. Brown CA, Sohani SB, Khan K, Lilford R, Mukhwana W. Antenatal care and perinatal outcomes in Kwale district, Kenya. BMC Pregnancy Childbirth. 2008:8:2.

15. Gupta S, Yamada G, Mpembeni R, Frumence G, Callaghan-Koru JA Stevenson R, et al. Factors associated with four or more antenatal care visits and its decline among pregnant women in Tanzania between 1999 and 2010. PLoS One. 2014;9. https://doi.org/10.1371/journal.pone.0101893. 
16. Pell C, Meñaca A, Were F, Afrah NA, Chatio S, Manda-Taylor L, et al. Factors affecting antenatal care attendance: results from qualitative studies in Ghana, Kenya and Malawi. PLoS One. 2013;8:e53747.

17. Chorongo D, Okinda FM, Kariuki EJ, Mulewa E, Ibinda F, Muhula S, et al. Factors influencing the utilization of focused antenatal care services in Malindi and Magarini sub-counties of Kilifi county, Kenya. Pan Afr Med J. 2016:ARTVOL. https://doi.org/10.11604/pamj.supp.2016.25.2.10520.

18. Koblinsky M, Moyer CA, Calvert C, Campbell J, Campbell OMR, Feigl AB, et al. Quality maternity care for every woman, everywhere: a call to action. Lancet. 2016. https://doi.org/10.1016/S0140-6736(16)31333-2.

19. Kruk ME, Gage AD, Arsenault C, Jordan K, Leslie HH, Roder-DeWan S, et al. High-quality health systems in the sustainable development goals era: time for a revolution. Lancet Glob Health. 2018;0. https://doi.org/10.1016/S2214-1 09X(18)30386-3.

20. Afulani PA. Rural/urban and socioeconomic differentials in quality of antenatal care in Ghana. PLoS One. 2015;10:e0117996.

21. Joshi C, Torvaldsen S, Hodgson R, Hayen A. Factors associated with the use and quality of antenatal care in Nepal: a population-based study using the demographic and health survey data. BMC Pregnancy Childbirth. 2014;14:94

22. Sharma J, Leslie HH, Kundu F, Kruk ME. Poor quality for poor women? Inequities in the quality of antenatal and delivery Care in Kenya. PLoS One. 2017;12:e0171236

23. Diamond-Smith N, Sudhinaraset M, Montagu D. Clinical and perceived quality of care for maternal, neonatal and antenatal care in Kenya and Namibia: the service provision assessment. Reprod Health. 2016;13:92.

24. Do M, Wang W, Hembling J, Ametepi P. Quality of antenatal care and client satisfaction in Kenya and Namibia. Int J Qual Health Care J Int Soc Qual Health Care. 2017;29:183-93.

25. Afulani PA, Sayi TS, Montagu D. Predictors of person-centered maternity care: the role of socioeconomic status, empowerment, and facility type. BMC Health Serv Res. 2018;18:360

26. Afulani PA, Diamond-Smith N, Golub G, Sudhinaraset M. Development of a tool to measure person-centered maternity care in developing settings: validation in a rural and urban Kenyan population. Reprod Health. 2017; 14:118.

27. MCD. Department of Health Services. 2016. http://migori.go.ke/index.php/ migori-county-departments/department-of-health. Accessed 2 Jun 2017.

28. Health Policy Project. Kenya County Health Fact Sheets. 2015. https://www. healthpolicyproject.com/index.cfm?id=kenyaCHFS. Accessed 2 Jun 2017.

29. Kenya National Bureau of Statistics, Ministry of Health, National AIDS Control Council, Kenya Medical Research Institute, National Council for Population and Development, Nairobi, Kenya, and The DHS Program, ICF International, Rockville, Maryland, USA. The DHS Program - Kenya: DHS, 2014 - Final Report (English). 2015. http://dhsprogram.com/publications/publication-FR3 08-DHS-Final-Reports.cfm. Accessed 18 Feb 2016.

30. Carr M. Women's economic empowerment: key to development. In: De Pauli $L$, editor. Women's empowerment and economic justice: reflecting on experience in Latin America and the Caribbean; 2000.

31. Stromquist NP. The theoretical and practical bases for empowerment. In: Women, education and empowerment: pathways towards autonomy. Carolyn Medel-Anonuevo; 1995.

32. Mosedale S. Assessing women's empowerment: towards a conceptual framework. J Int Dev. 2005;17:243-57.

33. Malhotra A, Schuler SR, Boender C. Measuring Women's empowerment as a variable in international development. 2002. https://landportal.info/library/2 015/09/measuring-women\%E2\%80\%99s-empowerment-variableinternational-development.

34. Jewkes R, Penn-Kekana L. Mistreatment of women in childbirth: time for action on this important dimension of violence against women. PLoS Med. 2015;12:e1001849.

35. Metrics for Measurement. EquityTool. M4MGMT. 2016. http://m4mgmt.org/ allprojects/the-equity-tool. Accessed 14 Jun 2017.

36. Afifi AA, Clark V, May S. Computer-aided multivariate analysis. 4th ed. Boca Raton: CRC Press; 2004

37. Doyle $C$, Lennox L, Bell D. A systematic review of evidence on the links between patient experience and clinical safety and effectiveness. BMJ Open. 2013;3. https://doi.org/10.1136/bmjopen-2012-001570.

38. Institute of Medicine (US) Committee on Quality of Health Care in America. Crossing the quality chasm: a new health system for the 21st century. Washington (DC): National Academies Press (US); 2001. http://www.ncbi. nlm.nih.gov/books/NBK222274/. Accessed 22 Dec 2017.
39. Bohren MA, Hunter EC, Munthe-Kaas HM, Souza JP, Vogel JP, Gülmezoglu AM. Facilitators and barriers to facility-based delivery in low- and middle-income countries: a qualitative evidence synthesis. Reprod Health. 2014;11:71.

40. Afulani PA, Phillips B, Aborigo RA, Moyer CA. Person-centred maternity care in low-income and middle-income countries: analysis of data from Kenya, Ghana, and India. Lancet Glob Health. 2019;7:e96-109.

41. Freedman LP, Ramsey K, Abuya T, Bellows B, Ndwiga C, Warren CE, et al. Defining disrespect and abuse of women in childbirth: a research, policy and rights agenda. Bull World Health Organ. 2014;92:915-7.

42. Dey A, Shakya HB, Chandurkar D, Kumar S, Das AK, Anthony J, et al. Discordance in self-report and observation data on mistreatment of women by providers during childbirth in Uttar Pradesh, India. Reprod Health. 2017; 14. https://doi.org/10.1186/s12978-017-0409-z.

43. Freedman LP, Kujawski SA, Mbuyita S, Kuwawenaruwa A, Kruk ME, Ramsey $K$, et al. Eye of the beholder? Observation versus self-report in the measurement of disrespect and abuse during facility-based childbirth. Reprod Health Matters. 2018;0:1-16.

44. Afulani PA, Kirumbi L, Lyndon A. What makes or mars the facility-based childbirth experience: thematic analysis of women's childbirth experiences in western Kenya. Reprod Health. 2017;14. https://doi.org/10.1186/s12978-01 7-0446-7.

45. Jamah A. How kickbacks for greedy doctors are making patients pay more. The standard. https:/www.standardmedia.co.ke/article/2000135631/how-kickbacksfor-greedy-doctors-are-making-patients-pay-more. Accessed 6 Oct 2018.

46. Boller C, Wyss K, Mtasiwa D, Tanner M. Quality and comparison of antenatal care in public and private providers in the United Republic of Tanzania. Bull World Health Organ. 2003;81:116-22.

47. Hutchinson PL, Do M, Agha S. Measuring client satisfaction and the quality of family planning services: a comparative analysis of public and private health facilities in Tanzania, Kenya and Ghana. BMC Health Serv Res. 2011; 11:203.

48. Kruk ME, Kujawski S, Mbaruku G, Ramsey K, Moyo W, Freedman LP. Disrespectful and abusive treatment during facility delivery in Tanzania: a facility and community survey. Health Policy Plan. 2014;33:e26-33.

\section{Publisher's Note}

Springer Nature remains neutral with regard to jurisdictional claims in published maps and institutional affiliations.
Ready to submit your research? Choose BMC and benefit from:

- fast, convenient online submission

- thorough peer review by experienced researchers in your field

- rapid publication on acceptance

- support for research data, including large and complex data types

- gold Open Access which fosters wider collaboration and increased citations

- maximum visibility for your research: over $100 \mathrm{M}$ website views per year

At BMC, research is always in progress.

Learn more biomedcentral.com/submissions 